\title{
Planck's Incostant
}

\author{
Fedele Lizzi*i \\ Dipartimento di Fisica "E. Pancini”, Università di Napoli "Federico II" \\ INFN, Sezione di Napoli, \\ Departament de Estructura i Constituents de la Matèria, Institut de Ciéncies del Cosmos, \\ Universitat de Barcelona. \\ E-mail: fedele.lizzi@na.infn.it
}

\section{Gianpiero Mangano}

INFN, Sezione di Napoli.

E-mail: mangano@na.infn.it

\section{Alberto Porzio}

CNR-SPIN, Unità di Napoli.

E-mail: porzio@na.infn.it

Is it possible that the fundamental Planck constant has stochastic fluctuations? We entertain this possibility in this talk, motived by a possible quantum structure of spacetime. We describe in which sense Panck's constant is inconstant (a nontrivial affair for fundamental dimensionful quantities. In our scheme $\hbar$ is time dependent, given by random fluctuations around zero, characterized by the correlation time scale $\tau$. As examples we briefly discuss possible experimental signatures using coherent states and interferometers.

Proceedings of the Corfu Summer Institute 2015 "School and Workshops on Elementary Particle Physics and Gravity"

1-27 September 2015

Corfu, Greece

\footnotetext{
* Speaker.

${ }^{\dagger}$ Work based on [1].
} 


\section{Quantum Spacetime and Noncommutativity}

There is a common belief that at the Planck's scale (or even before) something is happening... General Relativity and quantum field theory, in their present form, are not compatible. This is just a conceptual problem for all case in which their domain of compatibility do not overlap. One may use the latter to describe macroscopic black holes and galaxies, and the latter the collision of particles at $L H C$. The problem arises when one has to describe objects and energies for which both theories are needed. Although a full theory of quantum gravity is not known, it is very likely that it will alter the very geometry of spacetime, leading possibly to a noncommutative geometry [2], or some forms of loop quantum gravity [3]. Given that we do not have a theory of quantum gravity, it is nevertheless reasonable to surmise that, being a quantization of general relativity, it will involve the very structure of spacetime. On the other side, being a quantum theory, it will be described by operators, and it may have noncommutative features.

The modification of spacetime might take the form of nontrivial commutation relations for the position operator:

$$
\left[x^{i}, x^{j}\right]=\mathrm{i} \theta^{i j}
$$

where the dimensionful quantity $\theta$ may be a tensor [4] or a quantity coming from string theory [5, 6], but more in general it may be an arbitrary and nonconstant quantity. Another possibility is the case of the generalized uncertainty principle $[7,8]$ In this case the deformation is of phase space:

$$
\left[x^{i}, p_{j}\right]=\mathrm{i} \hbar\left(1+F_{j}^{i}\right)
$$

Further generalizations are possibile considering also noncommutativity of momenta:

$$
\left[p_{i}, p_{j}\right]=\mathrm{i} C_{i j}
$$

The case of $\theta, F$ and $C$ constant (non spacetime dependent) has been much studied in later years, usually considering the deformation of products, usually via the Grönewold.Moyal product $[9$, 10], although alternative products are possible. This kind of noncommutativity breaks Poincaré invariance, it retains translation invariance, and indeed all translationally invariant products share some of of the Moyal-Grönewold product, such as infrared/ultraviolet mixing [11]. It is possible that such a spacetime might have "quantum" symmetries (see for example [12] and references therein).

For totally arbitrary $\theta, F$ and $C$, a theorem by Kontsevich [13] ensures that it is always possible to formally define a product, but the construction may be exceedingly difficult, and is in any case a formal series in the noncommutativity parameters, which may create problems of convergence. Moreover the issue of Lorentz noninvariance remains. The presence of a tensor defines always (except for the trivial case of two dimensions) some preferred directions, which break the symmetry ${ }^{1}$

Apart from noncommutative spacetime, other visions of quantum spacetime point to a granularity of it, ie.e. to some structures appearing at Planckinan scale. This begs the question of the measurement of such a spacetime, we will try to address some of these points in the next section.

\footnotetext{
${ }^{1}$ In more sophisticated approaches [4] the operators do not break Lorentz invariance, but preferred directions are picked from the vacuum.
} 


\section{How is space(time) measured?}

Obviously this question has many layers, we will discuss it from an abstract point of view, namely we will not concern ourselves with the actual apparatus, but will concentrate on the mathematical process leading to the definition of the position in a quantum context. Our discussion will be in the context of non relativistic quantum mechanics. A treatment using quantum field theory and/or relativity is more ambitious, but not beyond reach.

In a quantum theory the starting point for any measurable quantity is the concept of observable.They are the selfadjoint part of an algebra of operators represented on a (typically infinite dimensional) Hilbert space. Obviously not to every selfadjoint operator corresponds a quantity with a physical meaning, but certainly every measurable quantity is described, in the quantum context, by such an operator. To describe the state (in a physical sense) in which a system is, we use the mathematically concept of state $^{2}$, of course the homonymy is not a coincidence! Mathematically a state $\phi$ of a physical system is a positive and of unit norm map from the algebra to complex numbers, in other words, for any operator it must be

$$
\phi\left(A^{\dagger} A\right) \geq 0 ; \phi(\mathbb{1})=1
$$

A pure state is a state that cannot be written as the convex sum of two other states, in other words, it is impossible to find two other states $\phi_{1}, \phi_{2}$ and a real number $\lambda \in[0,1]$ such that

$$
\phi=\lambda \phi_{1}+(1-\lambda) \phi_{2}
$$

If one considers as Hilbert space the space of square integrable functions on $\mathbb{R}^{n}$, and as algebra the one of bounded operators generated by position and momentum, the two operators $\mathbf{x}_{0}^{i}$ and $\mathbf{p}_{\mathbf{j}_{0}}$ represented in the usual way (the reason for the presence of the subscript will be clear in the following):

$$
\begin{aligned}
\mathbf{x}_{0}^{i} \psi(x) & =x^{i} \psi(x) \\
\mathbf{p}_{\mathbf{j}_{0}} \psi(x) & =-\mathrm{i} \hbar \frac{\partial}{\partial x^{j}} \psi(x)
\end{aligned}
$$

The pure states of this noncommutative algebra are the vectors of the Hilbert space, i.e. the pure states of quantum mechanics. the other states being a generic density matrix.

Alternatively it is possible to consider the algebra of continuous functions on $\mathbb{R}^{n}$ vanishing at infinity. This is a commutative algebra generated by just $\mathbf{x}_{0}$. To these functions correspond operators represented as usual as a multiplication by a function:

$$
\mathbf{f} \psi(x)=f(x) \psi(x)
$$

then the pure states $\delta_{\vec{y}}$ are in one to one correspondence to the points of $\vec{y} \in \mathbb{R}^{n}$, and they act as

$$
\delta_{\vec{y}}(\mathbf{f})=f(\vec{y})
$$

and there does not correspond to them a vectors (they are the Dirac's $\delta$ distribution).In this view configuration space emerges as the selfadjoint part of a commutative subalgebra. From this commutative algebra it is possible to reconstruct the topology of configuration space as the set of pure states of this commutative algebra. This is the Gelfand-Naimark-Moser, reconstruction theorem, see for example [15].

\footnotetext{
${ }^{2}$ This material is standard and is treated in many books, for a view close in spirit to this talk see for example [14, 15].
} 


\section{Generic Commutation Relations}

Rewrite (1.2) generically as

$$
\left[x^{i}, p_{j}\right]=i H_{j}^{i}
$$

and consider the nontrivial commutation relations (1.1) and (1.3). They break Lorentz (or rather rotational) invariance by picking two vectors in space at each point in space and time (in case they are nonconstant). There is no evidence for such a breaking. The natural scale for (1.1) is $\sqrt{\frac{\hbar G}{c^{3}}} \simeq$ $10^{-35} \mathrm{~m}$, the one for $H_{j}^{i}$ is $\hbar=6.6310^{-34} \mathrm{~J} \mathrm{sec}$, while the one for $C$ is $\sqrt{\frac{\hbar c^{3}}{G}} \simeq 6.5 \mathrm{Kg} \mathrm{m} / \mathrm{sec}$, which unlike the other two is not microscopic.

\section{Fast varying and stochastic commutators}

One way to recover, at a certain level, the lost invariance is to have $\theta$ and $C$ depend on space and/or time and to be a random variables, fast oscillating around zero. In this way, even if the theory is not formally invariant, no measurement made with coarse grained detectors can detect this noninvariance. The invariance is recovered in an effective way, as an average. This is the central point of this talk. It is most natural to imagine che the correlation length and time of a such a quantum space time be of Planckian nature. From what has been said in Sect. 2, in quantum mechanics, what counts is an algebra of observables, and the fact that within it one can recognize a commutative subalgebra defining configuration space

Considering all of the variables of space time as a single vector, $Y^{A}=\left\{x^{i}, p_{j}\right\}, A=1 \ldots 2 d$ the non trivial commutations described earlier can be stored into a single antisymmetric $2 d \times 2 d$ matrix

$$
\Omega=\left(\begin{array}{cc}
\theta^{i j} & H_{j}^{i} \\
-H_{j}^{i} & C_{i j}
\end{array}\right)
$$

It is always possible, at least locally, to put the matrix $\Omega$ in canonical form with a Darboux transformation to obtain

$$
\Omega^{\prime}=\left(\begin{array}{cc}
0 & H^{\prime i} \\
-H_{j}^{\prime i} & 0
\end{array}\right)
$$

with $H^{\prime}$ diagonal. This is just a change of variables involving linear combinations of position and momenta leading to the definition of the new variables $x^{\prime}, p^{\prime}$.with the modified commutation relations:

$$
\begin{aligned}
& {\left[x^{\prime i}, p_{j}^{\prime}\right]=H^{\prime i}{ }_{j}} \\
& {\left[x^{\prime i}, x^{\prime i}\right]=0} \\
& {\left[p_{i}^{\prime}, p_{j}^{\prime}\right]=0}
\end{aligned}
$$

In other word we have a canonical structure for which the commutation relation between position and momenta is a varying, stochastically changing quantity: a Planck' Inconstant.

Having fundamental constants of nature to be variables is not a new idea, it goes back at least to Dirac's large number hypothesis [16]. But usually the variability was over long (cosmological) 
time scales. The previous reasoning instead suggests an effective Panck constant with a variability over very short scales.

A comment about dimensionality. Planck's constant has dimensions, and its time dependence should be always related to some particular combinations of dimensionful quantities, depending on the adopted experimental method. Only for adimensional combinations of fundamental constants, such as the fine structure constant $\alpha$, it is meaningful to speak about spacetime variation unambiguously. The variability of fundamental constants is a delicate issue, a careful discussion of it can be found in e.g. [17]. We will see later that the stochastic nature of $\hbar$ is encoded in a dimensionful time parameter $\tau$ and observable effects for light propagation or a harmonic oscillator show up through the dimensionless combinations of $\tau$ and other dimensionful quantities related to the problem at hand.

\section{Simple one dimensional models}

In the following we will concentrate on simple one dimensional models and consider the variation of $\hbar$ to be only time dependent. It serves only to illustrated our idea in a semi-realistic situation. A more complete treatment is beyond the scope of this talk. Let us therefore express the commmutation relations as

$$
[\mathbf{x}, \mathbf{p}]=i \hbar(1+\varepsilon(t))
$$

The stochasticity is enforced by the requirement that $\varepsilon$ is a rapidly changing (in time) quantity which oscillates in a random way around a mean:

$$
\begin{aligned}
\overline{\varepsilon(t)} & =0 \\
\overline{\varepsilon(t) \varepsilon\left(t^{\prime}\right)} & =\tau \delta\left(t-t^{\prime}\right)
\end{aligned}
$$

Overline denotes the mean over the $\varepsilon$ probability distribution. The fluctuations are uncorrelated for time differences larger than a typical correlation time $\tau$.

The time evolution of an operator is given by its commutator with an Hamiltonian (apart form possible explicit dependence on time):

$$
\frac{d \mathbf{A}}{d t}=\frac{1}{\mathrm{i} \hbar}[\mathbf{A}, \mathbf{H}]+\frac{\partial \mathbf{A}}{\partial t}
$$

This time also the first term on the right hand side depends on time via $\hbar$, and de are assuming that the dependence on $\varepsilon$ is given by the commutator. This means that also the Poisson bracket, whose quantization gives the commutator, is also fluctuating. Defining the Poisson structure by normalizing the commutator to $\hbar(1+\varepsilon(t))$ cancel all effects and one obtains standard quantum mechanics. This is coherent with the view that the fluctuations are an effective way to take into account an underlying structure of quantum gravity.

We now need to represent $x$ and $p$ as operators reflecting the modified commutator

$$
\begin{gathered}
\mathbf{x} \psi(x)=A(t) x \psi(x)=A(t) \mathbf{x}_{0} \psi(x) \\
\mathbf{p} \psi(x)=-i \hbar B(t) \frac{d}{d x} \psi(x)=B(t) \mathbf{p}_{0} \psi(x)
\end{gathered}
$$


with $A(t) B(t)=1+\varepsilon(t)$, and $\mathbf{x}_{0}, \mathbf{p}_{0}$ the canonical pair of standard quantum mechanics introduced in (2.3).

We treat position and momentum on the same footing and choose

$$
A(t)=B(t)=\sqrt{1+\varepsilon(t)},
$$

Such an effective variable $\hbar$ will undoubtedly have consequences at several levels. The effects will depend on the scale $\tau$

In [1] we investigated two possible experimental signatures. Surely there will be many more, and we hope that other groups will explore other possibilities. We looked at

- Free particles and interferometric experiments.

- Harmonic Oscillators and coherent light

The Schrödinger equation for the free particle will show a time dependence via $\varepsilon(t)$

$$
i \hbar \frac{\partial}{\partial t} \psi=\frac{1}{2 m}(1+\varepsilon(t)) \mathbf{p}_{0}^{2} \psi
$$

It is possible to solve it for a plane wave of momentum $p_{0}$

$$
\psi_{p_{0}}(x, t)=\frac{1}{\sqrt{2 \pi}} \exp \left[i \frac{p_{0} x}{\hbar}-i \frac{p_{0}^{2}}{2 m \hbar}\left(t+\int_{0}^{t} \varepsilon\left(t^{\prime}\right) d t^{\prime}\right)\right]
$$

When measuring an observable in this scheme there are two averaging processes, conceptually distinct.

- Averaging over the time fluctuations of $\varepsilon$. We indicate this averaging process with : $\overline{\mathbf{A}}$. This means that repeating the experiment at different time gives different results, and one averages over them.

- The quantum mechanical averaging.The possible results of a measurement are the eigenvalues of the operator with a probability given by the state. This second average we indicate with $\langle\mathbf{A}\rangle$

In practice, we are considering $\tau$ to be very small, certainly smaller than the experimental time resolution. Then the two averaging coincide. Repeating the experiment samples both distribution. Nevertheless they are conceptually different, and we will keep the two notations

We considered first the motion of a free particle. For a gaussian peaked at $\bar{p}$ and variance $\delta^{2}$ we have

$$
\psi(x, t)=\int \frac{d p_{0}}{\sqrt{2 \pi}} \frac{1}{\left(\pi \delta^{2}\right)^{1 / 4}} e^{-\frac{\left(p_{0}-\bar{p}\right)^{2}}{2 \delta^{2}}+i p_{0} x / \hbar-i p_{0}^{2}\left(t+\int_{0}^{t} \varepsilon(t)\right) /(2 m \hbar)}
$$

The mean distance travelled by a particle is the usual

$$
\overline{\langle\mathbf{x}\rangle}_{\psi}(t)-\overline{\langle\mathbf{x}\rangle}_{\psi}(0)=\frac{\bar{p}}{m} t
$$


The effect of the variability of $\hbar$ shows up in the uncertainty, which can be calculated to be:

$$
(\Delta \mathbf{x})_{\psi}^{2}(t)-(\Delta \mathbf{x})_{\psi}^{2}(0)=\frac{\delta^{2}}{2 m^{2}} t^{2}+\frac{\bar{p}^{2}+\delta^{2} / 2}{m^{2}} \tau t
$$

The motion is like a Brownian motion with diffusion coefficient

$$
D=\frac{\bar{p}^{2}+\delta^{2} / 2}{2 m^{2}} \tau
$$

For $\delta \ll \bar{p}$, one can view $D$ as due to scatterings with mean free path $(\bar{p} / m) \tau$. The suggestive picture emerging is the presence of scattering over the quantum structure of spacetime

The usual spreading of the wave packet will dominate, but the effect can be enhanced for massless particles. In this case

$$
(\Delta \mathbf{x})_{\psi}^{2}(t)-(\Delta \mathbf{x})_{\psi}^{2}(0)=c^{2} \tau t
$$

It is in principle possible to measure this effect in a double slit experiment with a screen ad distance $L$ from the slits. The effect is a change $\delta t$, the time of travel, responsible for the interference. The variance of the variation is

$$
\overline{\delta t^{2}}=\tau t=\tau L / c
$$

with $t=L / c$ the time mean value.Tthis entails a change of interference pattern which can be explicitly calculated. For frequency $\omega$ and intensity $I$ at the mid-point on the screen we have

$$
I \propto \frac{1}{4}\left|e^{-i \omega\left(t+\delta t_{1}\right)}+e^{-i \omega\left(t+\delta t_{2}\right)}\right|^{2}=\frac{1}{2}\left(1+\cos \left[\omega\left(\delta t_{1}-\delta t_{2}\right)\right]\right)
$$

where $\delta t_{1,2}$ are the uncorrelated time shift along the two paths.In the standard case the two waves show a constructive interference. Here, averaging over $\delta t_{1,2}$ we have

$$
I \propto \frac{1}{2}\left(1+e^{-\omega^{2} \tau L / c}\right)
$$

For large $L, t$ the intensity behaves as the two waves were not interfering The relevant parameter here is $\omega^{2} \tau L / c \geq 1$. A very preliminary analysis puts a bound for the Virgo ot Ligo interferometers[18, $19]$, whose sensibility is bound by the shot noise, of

$$
\tau<10^{-10} \mathrm{GeV}^{-1} \hbar
$$

The second example is the harmonic oscillator, and coherent light states. For a one-dimensional harmonic oscillator with frequency $\omega$ and mass $m$ the Hamiltonian reads

$$
\mathbf{H}=\frac{1}{2 m}(1+\varepsilon) \mathbf{p}_{0}^{2}+\frac{m \omega^{2}}{2}(1+\varepsilon) \mathbf{x}_{0}^{2},
$$

the variability of $\hbar$ manifests itself as time dependent mass and frequency, $M=m /(1+\varepsilon)$ and $\Omega=\omega(1+\varepsilon),[20,21]$, with $M \Omega=m \omega$ a constant. The Hamiltonian depends on time via an overall multiplicative factor, as a consequence

$$
\left[\mathbf{H}(t), \mathbf{H}\left(t^{\prime}\right)\right]=0
$$


and the time evolution operator can be explicitly calculated with a Dyson series

$$
U(t)=\exp \left(-\frac{\mathrm{i}}{\hbar} \int_{0}^{t} H\left(t^{\prime}\right) d t^{\prime}\right)
$$

Proceeding as usual we have

$$
\mathbf{H}=\hbar \omega(1+\varepsilon)\left(\mathbf{a}^{\dagger} \mathbf{a}+\frac{1}{2}\right)
$$

with the standard creation and annihilation operator

$$
\begin{aligned}
\mathbf{a} & =\sqrt{\frac{m \omega}{2 \hbar}} \mathbf{x}_{0}+\mathrm{i} \frac{1}{\sqrt{2 m \hbar \omega}} \mathbf{p}_{0} \\
\mathbf{a}^{\dagger} & =\sqrt{\frac{m \omega}{2 \hbar}} \mathbf{x}_{0}-\mathrm{i} \frac{1}{\sqrt{2 m \hbar \omega}} \mathbf{p}_{0} \\
{\left[\mathbf{a}, \mathbf{a}^{\dagger}\right] } & =1
\end{aligned}
$$

The equation of motion for $\mathbf{a}$ is

$$
\frac{d \mathbf{a}(t)}{d t}=-i \omega(1+\varepsilon(t)) \mathbf{a}(t)
$$

which has the formal solution

$$
\mathbf{a}(t)=\mathbf{a}(0) e^{-i \omega t} \sum_{n} \frac{(-i \omega)^{n}}{n !} \int_{0}^{t} d t_{1} \ldots \int_{0}^{t} d t_{n} \varepsilon\left(t_{1}\right) \ldots \varepsilon\left(t_{n}\right) .
$$

Averaging over $\varepsilon(t)$ probability distribution and computing $n$-point correlation functions in terms of two-point correlation à la Wick gives

$$
\begin{aligned}
\overline{\mathbf{a}(t)} & =\mathbf{a}(0) e^{-i \omega t} \sum_{k} \frac{\left(-\omega^{2}\right)^{k}}{2 k !}(2 k-1) ! !(\tau t)^{k} \\
& =\mathbf{a}(0) e^{-i \omega t} \sum_{k} \frac{\left(-\omega^{2} \tau t\right)^{k}}{2^{k} k !}=\mathbf{a}(0) e^{-i \omega t} e^{-\omega^{2} \tau t / 2} .
\end{aligned}
$$

Apart from standard oscillatory term, evolution is exponentially damped on time-scales larger than the characteristic time $2\left(\omega^{2} \tau\right)^{-1}$.

Consider now a coherent state $|\lambda\rangle$ at time $t=0$. With no loss of generality we take $\lambda$ real. As discussed already, position/momentum measurements at some particular time amounts to measure $\mathbf{x}_{0}$ and $\mathbf{p}_{0}$. From equation (5.24) after averaging over $\varepsilon$ distribution

$$
\begin{aligned}
\overline{\langle\mathbf{x}}_{\lambda}(t) & =\sqrt{\frac{2 \hbar}{m \omega}} \lambda \cos (\omega t) e^{-\omega^{2} \tau t / 2}, \\
\overline{\langle\mathbf{p}}_{\lambda}(t) & =-\sqrt{2 \hbar m \omega} \lambda \sin (\omega t) e^{-\omega^{2} \tau t / 2}, \\
{\overline{\left\langle\mathbf{x}^{2}\right\rangle_{\lambda}}}_{\lambda}(t) & =\frac{\hbar}{m \omega}\left[\frac{1}{2}+\lambda^{2}\left(1+\cos (2 \omega t) e^{-\omega^{2} \tau t}\right)\right], \\
{\overline{\left\langle\mathbf{p}^{2}\right\rangle_{\lambda}}}_{\lambda}(t) & =m \hbar \omega\left[\frac{1}{2}+\lambda^{2}\left(1-\cos (2 \omega t) e^{-\omega^{2} \tau t}\right)\right],
\end{aligned}
$$


so that coherent states do not saturate the lower limit of Heisenberg relation as time flows, unless $\lambda=0$,

$$
\overline{\Delta \mathbf{x}}_{\lambda} \overline{\Delta \mathbf{p}}_{\lambda}=\frac{\hbar}{2}\left[1+2 \lambda^{2}\left(1-e^{-\omega^{2} \tau t}\right)\right] .
$$

This effect is in all similar to decoherence processes affecting the oscillator phase but leaving its energy unperturbed. We show position mean value, squared mean value and uncertainty in Figure 1. The growing behaviour of $\overline{\Delta \mathbf{x}^{2}} \lambda$ can be appreciated provided the state remains in a coherent configuration for times $t \omega \geq(\tau \omega)^{-1}$.

These features could be constrained using optical cavities. Excited by an external coherent beam, they can store coherence properties of electromagnetic field for long times, and consist in spatial confinement between two highly reflecting surfaces of a well defined propagation mode. Commonly used in laser/maser physics and in optical experiments where high spectral spatial purities are required (high resolution spectroscopy, interferometry, quantum optics, etc.), their confinement ability can be quantified, as for mechanical oscillators, in terms of quality factor $Q=\omega t_{c}$, the ratio between energy lost in a cycle to the energy stored in the cavity, with $\omega$ the frequency and $t_{c}$ the cavity decay time. Using supermirrors, at optical frequency, values of $Q \sim 10^{15}$ are accessible [22].

A single mode coherent state of an electromagnetic harmonic oscillator is a minimum uncertainty states for any pair of orthogonal field quadrature operators [23, 24], the analogue of position and momentum operators. In phase space this state is represented by a two dimensional Gaussian distribution with equal variances at all direction. To keep a stricter analogy with the harmonic oscillator discussed here, we define field quadratures as $\mathbf{X}=\sqrt{\hbar / 2}\left(\mathbf{a}+\mathbf{a}^{\dagger}\right)$ and $\mathbf{Y}=i \sqrt{\hbar / 2}\left(\mathbf{a}^{\dagger}-\mathbf{a}\right)$. Measurements of the uncertainty for a given quadrature of an electromagnetic mode can be obtained by a homodyne detector.

For a random $\hbar$, coherent configuration of radiation do not saturate the lower bound $\hbar / 2$ for $\Delta \mathbf{X} \Delta \mathbf{Y}$, which monotonically increases and is related to $|\lambda|^{2}$, the mean photon occupation number, see equation (5.27). Measurements in resonant cavities however, are limited by $t_{c}$. For $t>t_{c}$ the coherent electromagnetic field escapes from cavity due to unavoidable couplings to the external thermal bath, and the system evolves towards vacuum state $\lambda=0$. To account for this effect we modify equation (5.27) by introducing an exponentially damped $\lambda$

$$
\overline{\Delta \mathbf{X}}_{\lambda} \overline{\Delta \mathbf{Y}}_{\lambda}=\frac{\hbar}{2}\left[1+2 \lambda^{2} e^{-2 t / t_{c}}\left(1-e^{-\omega^{2} \tau t}\right)\right] .
$$

This approximation is satisfied if $t_{c}=Q / \omega$ is much larger than $\tau$, so that $\lambda$ adiabatically decays on $\tau$ time scales. The r.h.s. of equation (5.28) grows for small times, reaches a maximum at $t_{*}$

$$
\left.\overline{\Delta \mathbf{X}}_{\lambda} \overline{\Delta \mathbf{Y}}_{\lambda}\right|_{t_{*}}=\frac{\hbar}{2}\left[1+\lambda^{2} Q \omega \tau\left(\frac{2}{2+Q \omega \tau}\right)^{(2+Q \omega \tau) /(Q \omega \tau)}\right]
$$

and eventually decays towards the standard value $\hbar / 2$. For $Q \omega \tau \ll 1, t_{*} \simeq t_{c} / 2-Q^{2} \tau / 8$.

Consider one measures the uncertainty product with some error. In the standard approach, this product for a coherent state gives $\hbar / 2$, so is a way to determine Planck constant, with some uncertainty, $\hbar \pm \Delta \hbar$. If the time behaviour of equation (5.28) is undetected, in particular the peak at 


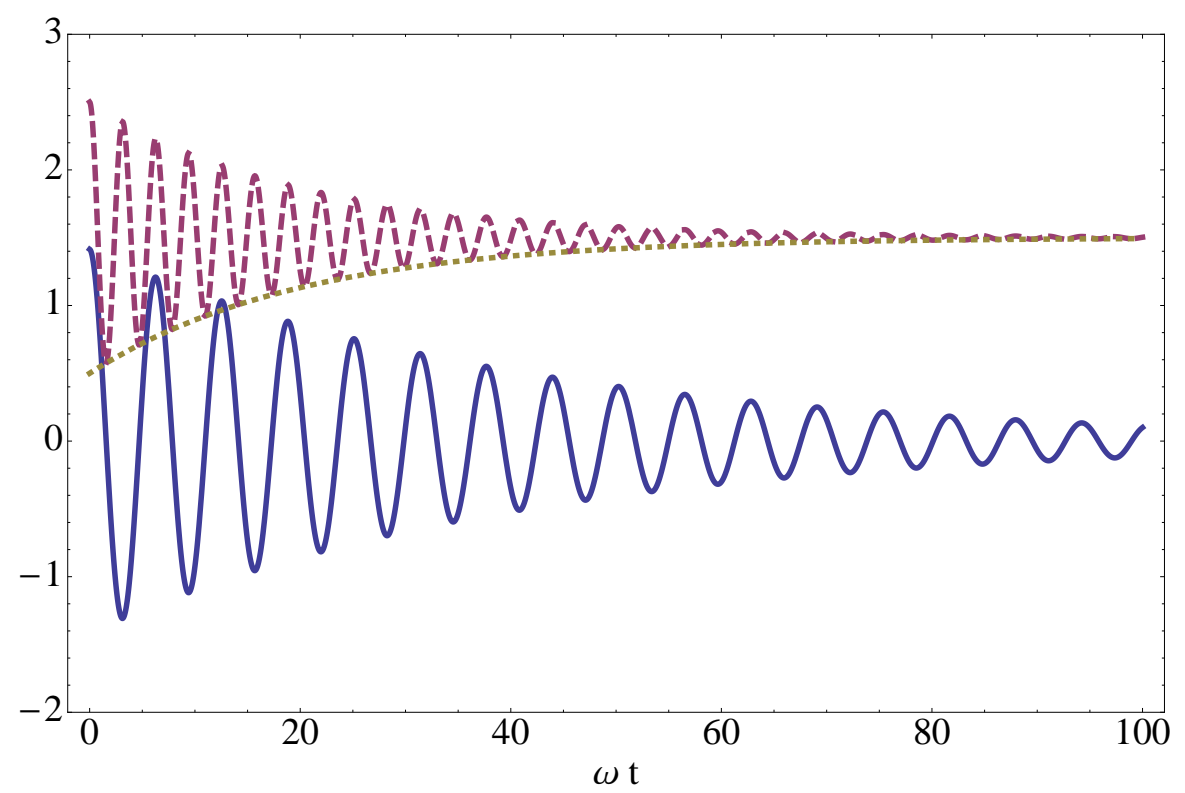

Figure 1: The time evolution of position mean value $\overline{\langle\mathbf{x}\rangle} \lambda$ (solid), $\overline{\left\langle\mathbf{x}^{2}\right\rangle_{\lambda}}$ (long-dashed) and squared uncertainty $\overline{\Delta \mathbf{x}^{2}}{ }_{\lambda}$ (short-dashed) for a coherent state with $\lambda=1$. Values are in units of appropriate powers of the length unit $\sqrt{\hbar /(m \omega)}$. We have chosen an unrealistic large value $\omega \tau=0.05$ to emphasize the non standard time behavior with respect to the case of a constant $\hbar$.

$t_{*}$, this means that $\tau$ should be sufficiently small

$$
\lambda^{2} Q \omega \tau\left(\frac{2}{2+Q \omega \tau}\right)^{(2+Q \omega \tau) /(Q \omega \tau)}<\frac{\Delta \hbar}{\hbar},
$$

To have an order of magnitude of this bound, we take $\lambda=1$. Choosing $Q=10^{15}, \omega \approx 3 \cdot 10^{15} \mathrm{~Hz}$, [22], and a measurement uncertainty $\Delta \hbar / \hbar \sim 1 \%$ we obtain

$$
\tau<10^{-32} \mathrm{~s},
$$

or, in terms of energy scale, $\Lambda=\hbar / \tau>10^{8} \mathrm{GeV}$. For smaller $\Delta \hbar$, the bounds on $\tau$ scales approximately as $(\Delta \hbar / \hbar) /(Q \omega)$, see equation (5.30).

\section{Discussion}

There are some elements, which are at the same time premises of this work, and that can have developments.

- Lorentz invariance may be the result of granular random structure on very short distances. This is not new (random lattices, causal sets), but it has not been used in noncommutative geometry, or to investigate some fundamental aspects of quantum mechanics.

- A quantum space time can give as effective theory, at low energy, one for which some constants of nature are actually variables. With a similar spirit, in a different development the 
possibility that Newton's gravitational constant is also a similarly varying quantity has been considered [25], and found to have interesting connections with a nonzero cosmological constant.

- In addition to particle, cosmological and astrophysical experiments, there could be signatures of quantum spacetime (non necessarily noncommutative) that can be seen using new kinds of experiments, such as interferometry, or optical cavities, which investigate the consequences of quantum gravity "granularity" also at a quantum mechanical level.

\section{Acknowledgments}

F.L. and G.M. are supported by INFN, I.S.'s GEOSYMQFT and TASP, respectively. This article is based upon work from COST Action MP1405 QSPACE, supported by COST (European Cooperation in Science and Technology). F.L. is partially supported by CUR Generalitat de Catalunya under projects FPA2013-46570 and 2014 SGR 104, MDM-2014-0369 of ICCUB (Unidad de Excelencia 'Maria de Maeztu').

\section{References}

[1] G. Mangano, F. Lizzi and A. Porzio, "Inconstant Planck's constant,” Int. J. Mod. Phys. A 30 (2015) no.34, 1550209 [arXiv:1509.02107 [quant-ph]].

[2] A. Connes, Noncommutative Geometry (Academic Press, 1994).

[3] C. Rovelli, "Loop Quantum Gravity”, Living Rev. Relativity 1 (1998), 1

[4] S. Doplicher, K. Fredenhagen and J. E. Roberts, "The Quantum structure of space-time at the Planck scale and quantum fields," Commun. Math. Phys. 172 (1995) 187 [hep-th/0303037].

[5] G. Landi, F. Lizzi and R. J. Szabo, "String geometry and the noncommutative torus," Commun. Math. Phys. 206 (1999) 603 [hep-th/9806099].

[6] N. Seiberg and E. Witten, "String theory and noncommutative geometry," JHEP 9909 (1999) 032 [hep-th/9908142].

[7] D. Amati, M. Ciafaloni and G. Veneziano, "Can Space-Time Be Probed Below the String Size?," Phys. Lett. B 216 (1989) 41.

[8] A. Kempf, G. Mangano and R. B. Mann, "Hilbert space representation of the minimal length uncertainty relation,” Phys. Rev. D 52 (1995) 1108 [hep-th/9412167].

[9] H. Grönewold, "On principles of quantum mechanics, Physica 12 (1946) 405.

[10] J. E. Moyal, "Quantum mechanics as a statistical theory," Proc. Cambridge Phil. Soc. 45, 99 (1949).

[11] S. Galluccio, F. Lizzi and P. Vitale, "Translation Invariance, Commutation Relations and Ultraviolet/Infrared Mixing," JHEP 0909 (2009) 054 [arXiv:0907.3640 [hep-th]].

[12] P. Aschieri, M. Dimitrijevic, P. Kulish, F. Lizzi and J. Wess, "Noncommutative spacetimes: Symmetries in noncommutative geometry and field theory," Lect. Notes Phys. 774 (2009) 1.

[13] M. Kontsevich, "Deformation quantization of Poisson manifolds. 1.," Lett. Math. Phys. 66 (2003) 157 [arXiv:q-alg/9709040 [q-alg]]. 
[14] J.M.G. Fell, R.S. Doran, Representations of *-Algebras, Locally Compact Groups and Banach *-Algebraic Bundles (Academic Press, 1988).

[15] J.M. Gracia-Bondia, J.C. Varilly, H. Figueroa, Elements of noncommutative geometry, Birkhauser, 2000.

[16] P.A.M. Dirac, “The Cosmological Constants”, Nature 139, 323 (1937).

[17] M. J. Duff, “How fundamental are fundamental constants?," Contemp. Phys. 56 (2015) no.1, 35 [arXiv:1412.2040 [hep-th]].

[18] A. Giazotto, “The Virgo Project: A Wide Band Antenna for Gravitational Wave Detection”, Nucl. Instrum. Meth. A 289, 518 (1990).

[19] A. Abramovici et al., "LIGO: The Laser interferometer gravitational wave observatory", Science 256, 325 (1992).

[20] P. Caldirola, "Forze non conservative nella meccanica quantistica", Nuovo. Cim. 18, 393 (1941).

[21] E. Kanai, “On the Quantization of the Dissipative Systems”, Prog. Theor. Phys. 3, 440 (1948).

[22] Young B.C., Cruz F.C., Itano W.M., and Bergquist J.C., Visible Lasers with Subhertz Linewidths. Phys. Rev. Lett., 82, 3799 (1999).

[23] E.C.G. Sudarshan, "Equivalence of semiclassical and quantum mechanical descriptions of statistical light beams", Phys. Rev. Lett. 10, 277 (1963).

[24] R.J. Glauber, "Coherent and incoherent states of radiation field”, Phys. Rev. 131, 2766 (1963).

[25] M. de Cesare, F. Lizzi and M. Sakellariadou, "Effective cosmological constant induced by stochastic fluctuations of Newton's constant," arXiv:1603.04170 [gr-qc]. 